\title{
Patchwork sequencing of tomato San Marzano and Vesuviano varieties highlights genome-wide variations
}

\author{
Maria Raffaella Ercolano ${ }^{1 *}$, Adriana Sacco ${ }^{1}$, Francesca Ferriello ${ }^{1}$, Raffaella D'Alessandro ${ }^{1}$, Paola Tononi ${ }^{2}$, \\ Alessandra Traini ${ }^{1,3}$, Amalia Barone ${ }^{1}$, Elisa Zago ${ }^{2}$, Maria Luisa Chiusano ${ }^{1}$, Genny Buson², Massimo Delledonne ${ }^{2}$ \\ and Luigi Frusciante ${ }^{1 *}$
}

\begin{abstract}
Background: Investigation of tomato genetic resources is a crucial issue for better straight evolution and genetic studies as well as tomato breeding strategies. Traditional Vesuviano and San Marzano varieties grown in Campania region (Southern Italy) are famous for their remarkable fruit quality. Owing to their economic and social importance is crucial to understand the genetic basis of their unique traits.

Results: Here, we present the draft genome sequences of tomato Vesuviano and San Marzano genome. A 40x genome coverage was obtained from a hybrid Illumina paired-end reads assembling that combines de novo assembly with iterative mapping to the reference S. Iycopersicum genome (SL2.40). Insertions, deletions and SNP variants were carefully measured. When assessed on the basis of the reference annotation, 30\% of protein-coding genes are predicted to have variants in both varieties. Copy genes number and gene location were assessed by mRNA transcripts mapping, showing a closer relationship of San Marzano with reference genome. Distinctive variations in key genes and transcription/regulation factors related to fruit quality have been revealed for both cultivars.

Conclusions: The effort performed highlighted varieties relationships and important variants in fruit key processes useful to dissect the path from sequence variant to phenotype.
\end{abstract}

Keywords: Combined assembling, Fruit quality, NGS sequencing, SNPs, Solanum lycopersicum

\section{Background}

Tomato (Solanum lycopersicum) is one of the most economically important vegetable crops worldwide. It is a rich source of micronutrients for human diet and a model species for fruit quality. Investigation of tomato genetic resources is a crucial issue for better straight evolution and genetic studies as well as tomato breeding strategies.

Since the late 18th and throughout the 19th and early 20th centuries a huge array of crosses and selection activities has taken place in Europe giving rise to a rich collection of tomato landraces [1,2]. In particular, an extensive selection work was performed in Italy by "Campania" farmers that developed several varieties adapted to local

\footnotetext{
*Correspondence: ercolano@unina.it; fruscian@unina.it

'Department of Agriculture Sciences, University of Naples Federico II, Via Universita' 100, 80055 Portici, Italy

Full list of author information is available at the end of the article
}

conditions and with quality requirements well delineated for specific uses. Among them, Vesuviano (RSV) and San Marzano (SM) varieties, grown in rich volcanic soil surrounding Vesuvius, are considered important models for fruit quality parameters. The Vesuviano has been cultivated on the Vesuvio hill, since the end of 19th century. It was selected by the local farmers because of its tolerance to the drought [3]. The origin of the San Marzano variety is very debatable. Some people report that San Marzano was a mutant from the local varieties (Corbarino); other people report that San Marzano was a natural hybridization between the grown varieties in the Agro-Sarnese-Nocerino area. Certainly, the cultivation of the San Marzano ecotype started in the years 1903-1904 in the Agro-Sarnese-Nocerino area becoming immediately a top variety for peeling [4]. Previous studies revealed that presently cultivated Vesuvio and 
San Marzano genotypes revealed peculiar sensory profiles in perception of sweetness and sourness [5,6]. San Marzano and Vesuvio fruits can purchased by at a price that is nearly five times higher than that of other varieties [7].

The advent of genomics era has brought a substantial increase in generation of data, knowledge and tools that can be employed in applied research. Candidate genes for important traits can be identified, and exploring functional nucleotide polymorphisms within genes of interest can facilitate breeders in combining favourable alleles. The decoding of the Heinz 1706 tomato reference genome SL2.40 will allow a better understanding of genetic basis of agronomic traits for developing novel genotypes $[8,9]$. Genome sequences and genomic tools offer exciting new perspectives and opportunities to track rates of sequence divergence over time, and provide hints about how genes evolve and generate new products by re-organization and shuffling of genomic sequences. Variant catalogues, however, will remain incomplete if forms of variation are undocumented. Good genome coverage is required to improve variant detection and accuracy and to study the polymorphism distribution across genomes. Genetic diversity studies have been improved by Next Generation Sequencing (NGS) based approaches [10,11]. However, interpreting the effect of genetic variation has typically relied on a reference genome. Indeed, alignment-consensus methods may have serious limitations in describing polymorphic regions and may also cause biases in interpreting the effect of variation on coding sequences. On the other hand de novo assembly approaches may theoretically overcome such problems, but pose a number of challenges due for example to repetitive sequences, low complexity sequences and closely related paralogs [12]. Alternative hybrid approaches can overcome limitations of alignmentconsensus methods $[13,14]$, allowing to capture a broader spectrum of sequence variation comparing genome with or without reference genome [15].

Here we describe the generation and analysis of San Marzano and Vesuviano tomato genome sequences. First, we reconstructed the genomes using a combination of iterative mapping and de novo assembly. Then, we annotated genes and documented the variation discovered, describing the typology and the distribution of variants between genotypes at chromosome level. Finally, as proof of concept we assessed the variability in fruit quality related genes, exploring the quantitative and qualitative impact of functional variants. Data produced can be helpful to investigate the genomic origins of phenotypic variation as well as to perform breeding programs.

\section{Methods}

\section{Sequencing}

A total amount of $2.5 \mu \mathrm{g}$ of genomic DNA was sonicated with Covaris S2 instrument to obtain 400 bp fragments.
DNA library preparation of SM and RSV tomato varieties (Additional file 1: Figure S1) was carried out using the TruSeq DNA Sample Prep Kit v2 (Illumina, San Diego, CA) accordingly to manufacturer instructions. RNA library preparation of SM and RSV tomato berry samples was carried out using the TruSeq RNA Sample Prep Kit v2 (Illumina, San Diego, CA) accordingly to manufacturer instructions.

Quality control of libraries was performed using High Sensitivity DNA Kit (Agilent, Wokingham, UK) and an accurate quantification was made using qPCR with KAPA Library Quantification kit (KapaBiosystems, USA). Libraries were then pooled and sequenced using Illumina Hiseq 1000 and applying standard Illumina protocols with TruSeq SBS Kit v3-HS and TruSeq PE Cluster Kit v3-cBot-HS kits (lllumina, USA). Libraries were sequenced with an Illumina Hiseq 1000 sequencer (Illumina Inc., San Diego, CA, USA) and 100-bp paired-end sequences were generated.

\section{Genome assembly and annotation transfer}

Genome reconstruction and variants identification were performed with the IMR/DENOM ver. 0.3.3 pipeline [14] using default parameters and the SL2.40 tomato genome [8] as reference. Repeats annotation was performed with RepeatMasker (v. open-3.3.0) using a custom redundant database available from SolGenomics website (ftp://ftp.solgenomics.net/tomato_genome/repeats/). ITAG 2.3 gene annotation was translated to the tomato reconstructed genomes by taking into account variants identified by IMR/DENOM pipeline and adjusting the coordinates accordingly using a custom software (http://ddlab.sci.univr. it/downloads/translate_coordinates.exe).

\section{Mapping of transcript sequences}

We independently mapped the 34,727 coding sequences (CDSs) [16,17] defined by the Solanum lycopersicum genome annotation to identify similarities versus RSV and SM tomato genomes using GenomeThreader [18], CDSs were also re-mapped versus SL2.40 to compare results between the three different genotypes. We filtered out alignments at similarity thresholds lower than $80 \%$ coverage and $90 \%$ identity. Correspondence among the loci in the three genotypes was defined on the basis of conserved loci position analyses at chromosome level and their distribution is reported using the CIRCOS program [19].

\section{Variants analysis and validation}

Identified variants between SL2.40 genome SM and RSV genotype were analysed using SnpEff version 2.1b (build 2012-04-20) [20] to predict their the effect on the genes in ITAG2.3 annotation. CDS non-synonymous variants were also submitted to PROVEAN (Protein Variation Effect Analyzer algorithm) analysis, which predicts the functional impact for all classes of protein sequence variations such 
as single amino acid substitutions but also insertions, deletions, and multiple substitutions [21]. To validate the identified SNPs, paired-end RNA-Seq reads (100 bp) from SM and RSV fruit samples were mapped against the reference genome SL2.40. SNPs were called using SAMtools 0.1 .18 [22] with a minimum read depth threshold of 6 and then compared with genomic reads using BEDTools 2.17.0 software [23].

\section{Enrichment analysis}

Our attention was focused on non-synonymous SNPs located in CDS belonging to four gene classes related to fruit quality (ascorbate biosynthesis; MEP/carotenoid pathway; ethylene-related genes; cell wall related genes); transcription factors and transcription regulators potentially involved in fruit ripening process. To evaluate if significant enrichment was present in specific metabolic pathways, an enrichment analysis based on Gene Ontology (GO) terms classification [24] was performed. We associated a GO term to each gene containing a non-synonymous coding variation running the BLAST2GO platform [25]. The data sets obtained were compared to the entire set of tomato genes with GO annotation (SOL Genomics. http://solgenomics.net/).

We performed a singular enrichment analysis (SEA) [26] which allows testing annotation terms against a list of interesting genes [27].

We used a hypergeometric test to compare each class to the reference background of genes. Hochberg (FDR) statistical correction was applied and a significance level of 0.05 was set. The minimum number of mapping entries was set as 1 to observe any significant enrichment. Only gene classes with a least 20 protein members (transcription factors, transcription regulators and cell wall) were subject to enrichment analysis.

\section{Data access}

All next-generation sequencing data are available in the Sequence Reads Archive (SRA) [SRA:SRP027562] Variants data in Snps, Deletions and Insertions (SDI) file format are available on SOL Genomics Network (SGN) website (ftp://ftp.solgenomics.net).

\section{Results}

\section{Genome assembly}

We sequenced Vesuviano (RSV) and San Marzano (SM) tomato varieties using Illumina $100 \mathrm{bp}$ paired-end reads with an insert size of about $250 \mathrm{bp}$. We obtained 155, 751,012 (X2) pareid-end reads for RSV and 177,758,218 (X2) paired-end reads for SM that, considering an expected size of about $900 \mathrm{Mb}$ [8], correspond to an average expected depth of about 34.6x and 39.5x genome equivalent, respectively (Additional file 2: Table S1). We chose to use a genome reconstruction method based on a combination of iterative read mapping against the tomato reference genome and de novo assembly that is able to describe complex loci on a single pass alignment [13] (Additional file 3: Figure S2). A similar number of mobile elements (63\%) and outstanding proportion of LTR elements (93\% of occupied length) with SL2.40 genome was found (Additional file 2: Table S2).

The size of the assembled genomes is very similar $(99.8 \%)$ to the reference genome (Additional file 2: Table S3). The slightly lower size observed in the reconstructed genomes may be related to a low efficiency of the method in detecting long insertions. We aligned the reads to the final assemblies to detect regions with a low read coverage, which may correspond to complex polymorphisms. The average N50 length of contiguous regions between polymorphic regions was of 77.5 Kbp and 72.7 Kbp for RSV and SM, respectively, while polymorphic regions sizes had a maximum of $88.7 \mathrm{Kbp}$ in RSV and with an average size of $1.4 \mathrm{Kbp}$ in both varieties (Additional file 2: Table S4). These polymorphic regions insist on 368 genes for the RSV and 328 genes for SM, and 283 of the genes interested by polymorphic regions are in common between the two varieties. Interestingly, SNPs distribution on regions of chromosome 9 and 11, implicated in the introgression of S.pimpinellifolium disease resistance loci into "SL2.40", showed higher density than average (Additional file 4: Figure S3), confirming previous findings. [8]. We detected 206,867 and 177,179 single base variants compared to the reference genome for RSV and SM respectively, of these 3,343 were shared between the two genotypes (Table 1). A small fraction (3.3\% in average) of the single base variants was ambiguous and, most probably, corresponded to heterozygous variants or misalignments due to repeated sequences. In fact, $61 \%$ (RSV) and 63\% (SM) of the putative heterozygous variants in either cultivar were located in annotated repeats and are most probably an artefact. We also identified a fairly large number of indels or unbalanced insertions (258,023 in global considering both varieties). Most SNPs were detected in intergenic and intronic regions (Table 2); as a whole the SNPs affected 23,220 and

Table 1 Variants statistics

\begin{tabular}{lccc}
\hline & RSV & SM & Common \\
\hline SNPs & $206,867(199,502)$ & $177,179(169,704)$ & $3,343(3,160)$ \\
Deletions & 46,433 & 44,561 & 25,208 \\
Insertions & 77,882 & 75,984 & 55,668 \\
Unbalanced & 6,537 & 6,626 & 1,343 \\
insertions & & & \\
Total & $\mathbf{3 3 7 , 7 1 9}$ & $\mathbf{3 0 4 , 3 5 0}$ & $\mathbf{8 5 , 5 6 2}$
\end{tabular}

Number of sequence variants in Vesuviano (RSV) and San Marzano (SM) varieties compared to the reference genome (SL240) In brackets is reported the number of unambiguous SNPs In the last column the number of variants in common between the two varieties is shown. 
Table 2 Counts of identified SNPs and of genes affected by them

\begin{tabular}{|c|c|c|c|c|}
\hline & \multicolumn{2}{|c|}{ RSV } & \multicolumn{2}{|c|}{ SM } \\
\hline & SNPs & Genes & SNPs & Genes \\
\hline Intergenic & $188,769(181,758)$ & $16,705(16,247)$ & $158,964(151,971)$ & $14,530(13,939)$ \\
\hline Intronic & $11,907(11,766)$ & $2,880(2,836)$ & $12,232(11,979)$ & $2,576(2,490)$ \\
\hline UTR & $624(610)$ & $381(376)$ & $686(671)$ & $361(353)$ \\
\hline CDS & $5,567(5,368)$ & $3,254(3,132)$ & $5,297(5,083)$ & $2,886(2,752)$ \\
\hline Total & $206,867(199,502)$ & $23,220(22,591)$ & $177,179(169,704)$ & $20,353(19,534)$ \\
\hline
\end{tabular}

Results are given distinguishing in which region the SNPs are found, if they are in intergenic regions, in genes, in exonic regions or in the coding regions of genes using the ITAG23 annotation of reference genome For variants that insists on a gene, a count of affected genes is also given In brackets are given the numbers of the unambiguous SNPs.

20,353 genes for RSV and SM, respectively. Comparison with RNA-Seq reads from RSV and SM samples showed that approximately $90 \%$ of the SNPs covered with a minimum read depth of 6 were validated (data not shown).

Indels sizes varied from single base up to $6,011 \mathrm{bp}$ in the case of insertions and 36,162 bp in the case of deletions. While the majority of the indels were shorter than 6 bases, we detected 105 insertions longer than 100 bp in RSV affecting 62 genes and 97 in SM affecting 60 genes (Table 3). Moreover, we detected 2,499 deletions longer than $100 \mathrm{bp}$ in RSV affecting 1,081 genes and 2,461 in SM affecting 1,042 genes (Table 4, Additional file 2: Table S4). However, we noticed that while SNPs were mostly specific of each cultivar, most of the insertions (71.4\% of RSV insertions; $73.3 \%$ of SM insertions) and deletions (54.3\% of RSV deletions; $56.6 \%$ of SM deletions) detected in each variety were shared with the other genotype (Table 1) and occurred with an average frequency of 1 indel every $6 \mathrm{~Kb}$. These findings resemble quite closely the frequency of estimated indel error rates

Table 3 Insertion statistics

\begin{tabular}{|c|c|c|c|c|c|}
\hline \multicolumn{6}{|c|}{ Insertions } \\
\hline & \multirow[t]{2}{*}{ Feature } & \multicolumn{2}{|c|}{ RSV } & \multicolumn{2}{|c|}{ SM } \\
\hline & & Variants & Genes & Variants & Genes \\
\hline \multirow[t]{4}{*}{ Total } & Intergenic & 69,615 & 18,730 & 67,850 & 18,432 \\
\hline & Intronic & 7,187 & 4,069 & 7,096 & 4,010 \\
\hline & UTR & 557 & 485 & 567 & 489 \\
\hline & CDS & 523 & 418 & 471 & 400 \\
\hline \multirow[t]{4}{*}{ Length $<6$} & Intergenic & 63,307 & 18,006 & 62,241 & 17,736 \\
\hline & Intronic & 6,846 & 3,975 & 6,791 & 3,929 \\
\hline & UTR & 535 & 466 & 553 & 478 \\
\hline & CDS & 381 & 321 & 362 & 315 \\
\hline \multirow[t]{4}{*}{ Length > 100} & Intergenic & 97 & 54 & 91 & 54 \\
\hline & Intronic & 4 & 4 & 2 & 2 \\
\hline & UTR & 0 & 0 & 0 & 0 \\
\hline & CDS & 4 & 4 & 4 & 4 \\
\hline
\end{tabular}

Statistics of the number of insertions and the number of corresponding annotated genes. reported for the reference genome SL2.40 (1 every 6.4 Kbp) [8]; and suggest that common indels may be due to errors in the reference genome rather than to true indels.

\section{Gene annotation}

We took advantage of the existing high quality Solanum lycopersicum reference annotation (ITAG2.3) released by the International Tomato Genome Sequencing Consortium [8] to annotate the assembled genomes for the RSV and SM varieties. The original annotations were transferred taking into account the cumulative effect of insertions and deletions along the whole length of the chromosomes. In order to evaluate the reliability of the transferred annotations we analysed the potential effect of detected variants projecting them on the corresponding protein coding sequences in each variety. Most variants were located outside the gene loci with only a smaller fraction harbouring SNPs or indels inside their coding sequences (Tables 2, 3 and 4). In particular, we found that most of the proteins encoded by RSV (98.1\%)

Table 4 Deletion statistics

\begin{tabular}{|c|c|c|c|c|c|}
\hline \multicolumn{6}{|c|}{ Deletions } \\
\hline & \multirow[t]{2}{*}{ Feature } & \multicolumn{2}{|c|}{ RSV } & \multicolumn{2}{|c|}{ SM } \\
\hline & & Variants & Genes & Variants & Genes \\
\hline \multirow[t]{4}{*}{ Total } & Intergenic & 43,385 & 9,909 & 41,604 & 9459 \\
\hline & Intronic & 2326 & 1384 & 2317 & 1354 \\
\hline & UTR & 141 & 110 & 147 & 115 \\
\hline & CDS & 581 & 472 & 493 & 404 \\
\hline \multirow[t]{4}{*}{ Length $<6$} & Intergenic & 34,567 & 8103 & 32,974 & 7696 \\
\hline & Intronic & 1898 & 1190 & 1914 & 1162 \\
\hline & UTR & 114 & 88 & 114 & 87 \\
\hline & CDS & 369 & 296 & 331 & 257 \\
\hline \multirow[t]{4}{*}{ Length > 100} & Intergenic & 2365 & 950 & 2337 & 920 \\
\hline & Intronic & 80 & 77 & 80 & 78 \\
\hline & UTR & 6 & 6 & 7 & 7 \\
\hline & CDS & 48 & 48 & 37 & 37 \\
\hline
\end{tabular}

Statistics of the number of deletions and the number of corresponding annotated gene. 


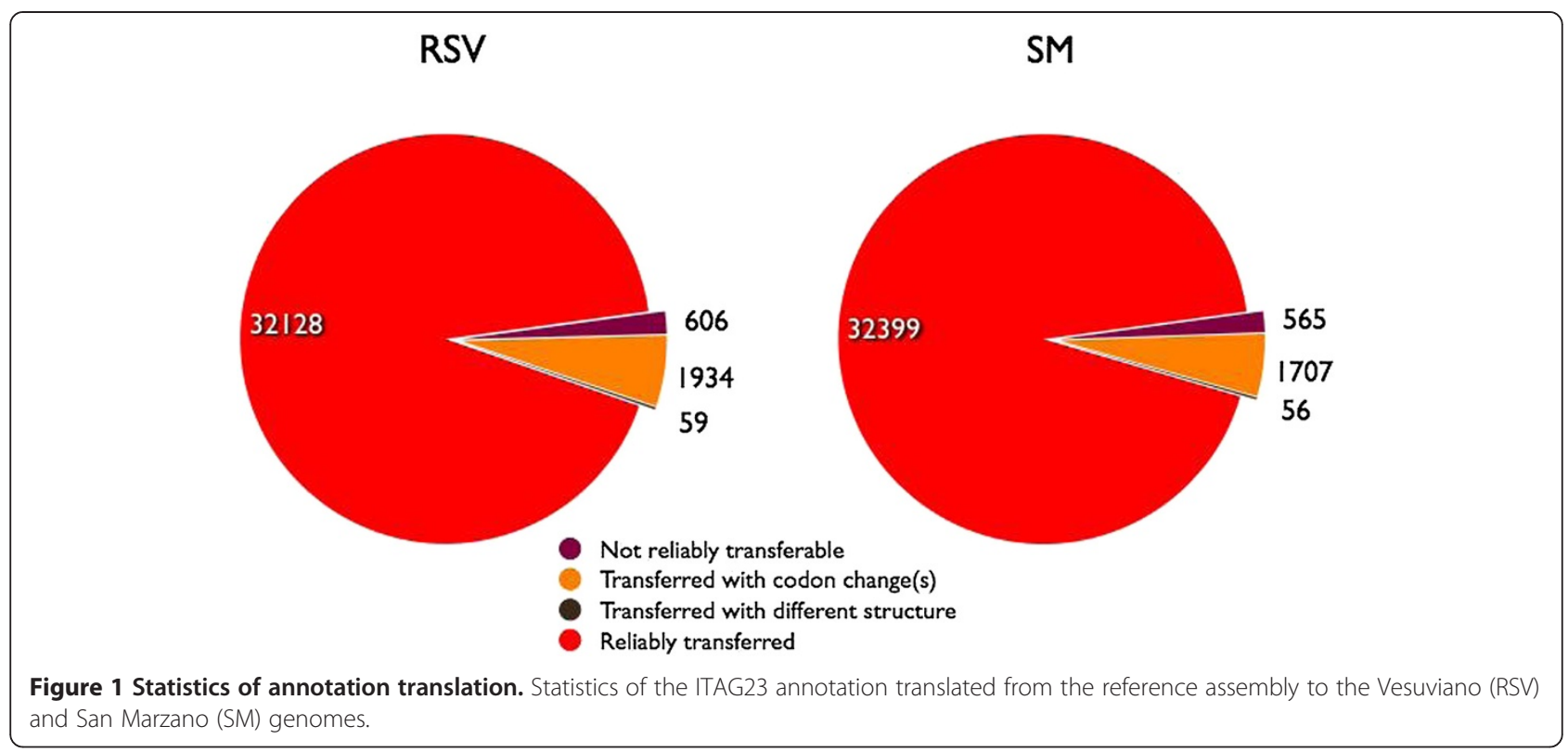

and SM genes (98.2\%) were not affected by potentially disrupting mutations and were reliably transferred to the corresponding genomes (Figure 1). A small number of annotations $(\approx 0.1 \%)$ was predicted to have an altered gene structure due to mutations in splice sites and were classified as "transferred with putative altered structure". Moreover, 606 RSV and 565 SM genes, corresponding respectively to $1.7 \%$ and $1.6 \%$ of the total annotations, were predicted to be potentially affected by disrupting mutations such as frameshifts and alteration of the start or stop codon and could not be reliably transferred (Figure 1 and Additional file 2: Table S5).

\section{Transcript sequences mapping}

We also mapped the 34,727 CDSs defined by the Solanum lycopersicum genome versus RSV, SM and the SL2.40 reference genomes. As expected, since the analyses was conducted at coding sequence level, better highlighted similarities between loci coding for the same protein family, and also some CDSs mapped more than one time along the three different genotypes (Table 5). Figure 2 reported the distribution of these loci over the SL2.40 genome. The distribution of S. lycopersicum genes for which CDSs are not

Table 5 Number of tomato genes mapped versus the reference tomato (SL240), the Vesuviano (RSV) and San Marzano (SM) genomes

\begin{tabular}{lccc}
\hline & SL240 & RSV & SM \\
\hline 1 match & 29,844 & 29,940 & 29,934 \\
$>1$ match & 4,755 & 4,621 & 4,638 \\
total & 34,600 & 34,561 & 34,572 \\
\hline
\end{tabular}

The number of genes resulting in one match or in multiple matches are indicated. mapped in SM and RSV, are highlighted by coloured lines over the grey chromosome bars. The gene loci, resulting from the mapping procedure, were compared among the twelve chromosomes of each genotype. This permitted to define any difference at genome level that could be associated to an unsuccessful mapping of CDS sequences, pointing out variability of gene loci distribution, of their protein coding exon-intron organization, or at nucleotide level, detectable thanks to difference of similarity score between the genotypes. Figure 3 reported a Venn diagram indicating the number of loci that shared the same relative position in the three genomes, and those that are present only in two or even one of the genotypes. In SL2.40 and SM genomes 243 loci maintained the same position, while in SL2.40 and RSV only 204. Among the 54,517 loci that are in common between the three different genotypes, 3,411 in total showed different similarity scores (in terms of percentage of identity and coverage of the aligned mRNA versus the genome) when compared with the reference genome loci organization. Specifically, RSV showed differences in 2,224 loci, while SM showed difference in 1,610 loci. Moreover, when counting the number of loci with identical similarity score, 881 loci from SM are identical to SL2.40 ones, while 596 genes in RSV resulted identical to SL2.40. These evidences suggest a higher similarity between SL2.40 and SM genomes.

Analysis of genetic variants in fruit quality related genes The analysis of genetic differences between RSV and SM genomes and the reference tomato genome SL2.40 has been focused on four gene classes related to fruit quality (ascorbate biosynthesis; MEP/carotenoid pathway; ethylene-related genes; cell wall related genes); transcription 


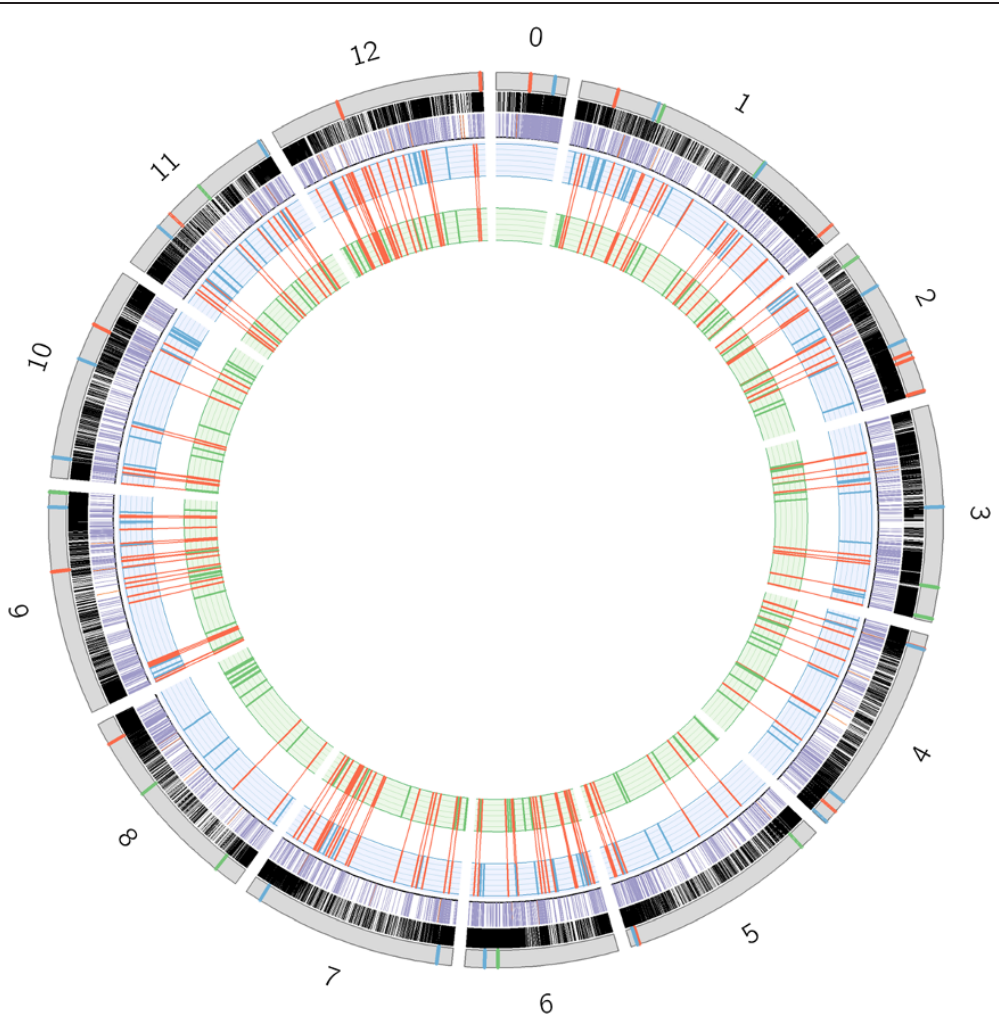

Figure 2 Main results from ITAG23 gene mapping on SL240, Vesuviano (RSV) and San Marzano (SM) tomato genomes. Grey bars represent the twelve tomato chromosomes and chromosome 0 The distribution of the genes within the SL240 genome is shown by black lines The distribution of genes mapped more than once is shown in the following circle with purple lines when the redundancy is equal or lower than 30 copies and with orange lines when genes re-mapped more than 30 times SM and RSV specific behaviours are indicated in green and in blue, respectively, in the whole picture, while the common behaviours among the two genotypes are indicated in red In particular, on each chromosome (grey bars) colored lines indicate the distribution of the S lycopersicum genes which are not mapped in the two SM and RSV genotypes The two inner circles indicate the distribution of genes that are specific to RSV (circle with a light blue background) and to SM (circle with a light green background) genotypes, along the respective pseudomolecules Common genes identified exclusively in chromosome positions from the two newly sequenced genotypes are in red and are linked by red connectors between the two inner circles to better highlight the conserved positions of these genes in the two newly sequenced genomes

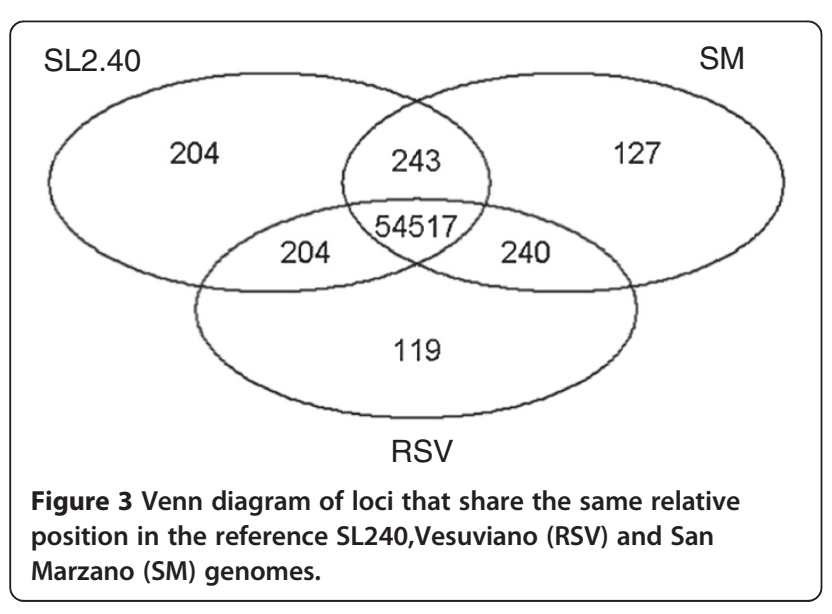

factors and transcription regulators potentially involved in fruit ripening process were also included (Table 6). A high percentage of genes belonging to all investigated classes showed variants. On average 10 variations for gene have been identified, ranging from 7 to 14 in RSV and from 7 to 19 in SM. The total number of varied genes is not indicative of specificity of variants for RSV or SM genes. Table 7 indicated that RVS and SM have 2,566 common genes with variants; nevertheless specific varied genes for each variety were also highlighted. Indeed, RSV showed a higher percentage of specific polymorphism (5.6\%) compared to SM (2.9\%). Interestingly, three ACS genes and a ETR1 gene involved in ethylene biosynthesis varied only in RSV (Additional file 2: Table S6). A high percentage of variations is included in upstream and downstream regions, with values (on total variants belonging to each class) ranging between $23.60 \%$ and $61.80 \%$ for RSV tomato and from $23.75 \%$ and $61.25 \%$ for 
Table 6 Number and percentage of polymorphic genes and number of variants identified for each fruit quality-related class of genes in Vesuviano (RSV) and San Marzano (SM) varieties Data are referred to the tomato reference genome (SL240)

\begin{tabular}{|c|c|c|c|c|c|c|c|}
\hline \multirow{3}{*}{ Gene class } & \multirow{3}{*}{ Genes no } & \multicolumn{3}{|c|}{ RSV } & \multicolumn{3}{|c|}{ SM } \\
\hline & & \multicolumn{2}{|c|}{ Polymorphic genes } & \multirow{2}{*}{$\begin{array}{c}\text { Variants } \\
\text { No }\end{array}$} & \multicolumn{2}{|c|}{ Polymorphic genes } & \multirow{2}{*}{$\begin{array}{c}\text { Variants } \\
\text { No }\end{array}$} \\
\hline & & No & $\%$ & & No & $\%$ & \\
\hline Ascorbate biosynthesis & 23 & 18 & 783 & 265 & 18 & 783 & 349 \\
\hline MEP/carotenoid pathway & 46 & 35 & 761 & 337 & 34 & 739 & 351 \\
\hline Ethylene-related genes & 52 & 48 & 923 & 466 & 44 & 846 & 448 \\
\hline Cell wall & 718 & 589 & 820 & 5,303 & 562 & 783 & 4,471 \\
\hline Transcription factors & 2,025 & 1,710 & 844 & 17,215 & 1,662 & 821 & 15,412 \\
\hline Transcription regulators & 434 & 353 & 813 & 2,660 & 344 & 793 & 2,496 \\
\hline Total & 3,298 & 2,753 & & 26,246 & 2,664 & & 23,527 \\
\hline
\end{tabular}

SM tomato (Figure 4). Putative impact of variants has been evaluated, focusing on non-synonymous variants localized in the coding sequence (CDS). The number of genes with non-synonymous variants in the CDS found specifically in RSV or in SM and the number of common variants are reported in Additional file 2: Table S7. In order to understand if an amino acid substitution has an impact on the biological protein function these gene subsets have been analyzed using the PROVEAN predictor. Out a total of 386 genes analysed belonging to the selected groups, 45 showed predicted deleterious non-synonymous variations $(11.6 \%)$ in the coding sequence when translated as amino acid substitutions (Table 8). The transcription factor class showed the highest number of deleterious substitution with 5, 6, and 11 genes in SM, RSV, and both genotypes, respectively. Similarly, deleterious variations for the protein function were observed in genes belonging to the cell wall and transcription regulators categories. Moreover, to highlight if there were gene functional categories susceptible of significant variation among the two tomato varieties and the reference, a singular enrichment analysis (SEA) was performed. The analyses showed that the gene

Table 7 Common and specific genes related to fruit quality with variants in Vesuviano (RSV) and San Marzano (SM) varieties

\begin{tabular}{lccc}
\hline Gene class & $\begin{array}{c}\text { Common } \\
\text { No }\end{array}$ & RSV & SM \\
& 16 & 2 & No \\
\hline Ascorbate biosynthesis & 33 & 2 & 2 \\
MEP/carotenoid & & 4 & 1 \\
pathway & 44 & 110 & 0 \\
Ethylene-related genes & 1,600 & 25 & 62 \\
Cell wall & 328 & 44 & 16 \\
Transcription factors & 545 & $\mathbf{1 8 7}$ & $\mathbf{9 8}$ \\
Transcription regulators & $\mathbf{2 , 5 6 6}$ & & \\
Total & & & \\
\hline
\end{tabular}

class of transcription factors with non-synonymous variants common to both tomato varieties was enriched for three molecular function GO terms: interleukin-6 receptor binding (GO:0005138), cytokine activity (GO:0005125) and RNA polymerase II transcription elongation factor activity (GO:0016944). RSV transcription regulation variants showed enrichment in molecular GO function for ethylene-binding class (GO:0051740), due to the presence of the gene encoding for the ethylene receptor (Solyc 07g056580 histidine kinase-related protein, a variant absent in SM). RSV transcription regulation non-synony mous variants showed enrichment in tight junction class (GO:0005923) because of the presence of the SNF2 helicase gene (Solyc03g095680, histone linker, a variant absent in SM). Figure 5 reports common and variety-specific non-synonymous cell wall coding variants enriched classes. Common non-synonynous variants showed enrichment in molecular function GO terms corresponding to hydrolase activity, hydrolyzing O-glycosyl compounds (GO:0004553), galactosidase activity (GO:0015925), coniferin betaglucosidase activity (GO:0047782), beta-galactosidase activity (GO:0004565). RSV-specific non-synonymous variants were enriched in fucosyltransferase activity (GO:00 08417) and polygalacturonate 4-alpha-galacturonosyltrans ferase activity (GO:0047262). Indeed, the presence of nonsynonymous variants in the fucosyltransferase 7 gene (Soly c03g115830) and in the glycosyltransferase (Solyc07g05 5930) determined a private significant enrichment in those functional classes.

\section{Discussion}

In this work the tomato RVS and SM genomes have been sequenced and assembled using a strategy based on iterative mapping and de novo assembly [14]. This method showed to be less demanding in terms of sequencing depth and multiple libraries construction compared to a complete de novo assembly. The catalogue of tomato 
A)

\section{RSV variation classes}

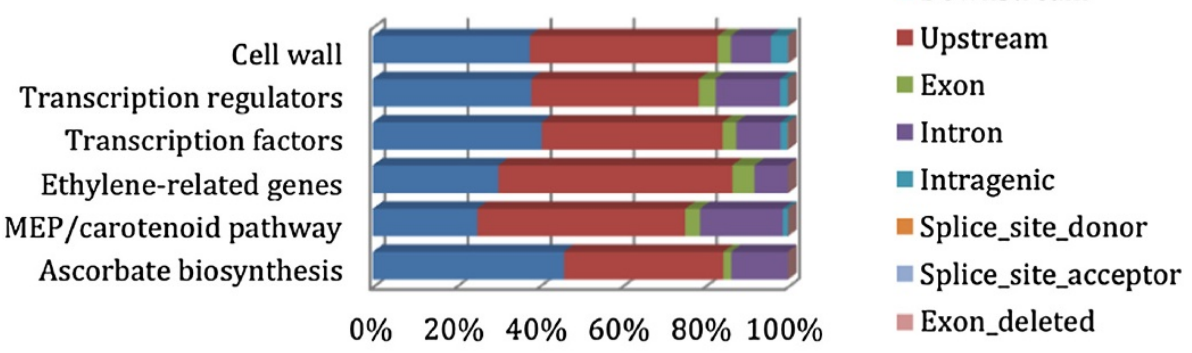

B)

\section{SM variation classes}

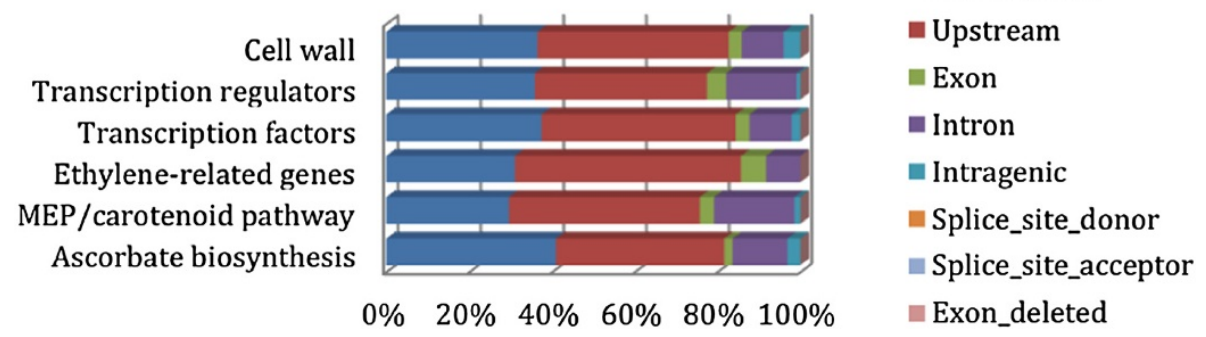

Figure 4 Classification of variants in fruit quality and ripening related genes. Classifications of variants were based on the their gene location A) Vesuviano (RSV) sequence variants B) San Marzano (SM) sequence variants.

genetic variants produced using this valuable approach allowed enlarging the list available (http://solgenomics. net/search/markers) with a relative low investment. The magnitude of the number of variants found is not comparable with earlier catalogue, based on transcriptome sequencing or oligonucleotide arrays [28-32]. In addition, other types of variations in CDS sequences were evidenced.

The chromosome pseudomolecules obtained allowed studying with high accuracy genome colinearity useful for gene mapping and marker-assisted breeding. At 40x sequence coverage, we estimated that approximately $99 \%$ of the tomato reference genome could be genotyped. Our analysis produced approximately 200,000 SNPs and more than 130,000 indels. In accordance with the high level of homozygosis reported for tomato cultivars [29], a small fraction (approximately 3\%) of heterozygous variants or sequence misalignments were identified in either cultivar. Variation in the level of polymorphism among chromosomes was found. Indeed, the chromosome variation could reflect selection history rather than polymorphism discovery [30]. More than 3,000 genes in both varieties showed different similarity values at exon level when compared with reference genome. A slight higher colinearity between SM and the reference genome was found, suggesting a their closer relationship. Genome-wide structural and gene content variations are hypothesized to drive important phenotypic variation within a species [33]. However, in most cases deletions are common to both varieties and their frequency is consistent with previous data on indel errors in the reference genome, and thus we suspect that a percentage of de novo assembled sequences corresponds to sequences missing from the reference genome.

Based on the tomato gene model set, a limited number of altered genes were detected in each variety, while 1,934 RSV and 1,707 SM transferred annotations were affected by mutations potentially causing amino acidic substitutions of unknown effect on the protein function. A subset of these SNPs was restricted to a single variety. The study of distribution of variants across the genomes of the sequenced variety is important. Number, location and predicted effects can gain insights in plant diversification. Indeed, the selective forces acting over time on diverse traits could have driven the fixation of positive mutations in each variety. Whether a polymorphic equilibrium is reached depends on the intensity of selection and the relative distances to the optimum of the homozygosis at each locus [34]. 
Table 8 Fruit quality related genes affected by deleterious mutation in SM, RSV or both varieties

\begin{tabular}{|c|c|c|c|}
\hline \multicolumn{4}{|l|}{ SM } \\
\hline Gene & Annotation & Variant & Score value \\
\hline \multicolumn{4}{|l|}{ Transcription factors } \\
\hline Solyc01g1062302 & B3 domain-containing protein & V79F & -3233 \\
\hline Solyc03g1182902 & Auxin response factor 2 & S220G & -3818 \\
\hline Solyc04g0094402 & NAC domain protein & Y212C & -3401 \\
\hline Solyc04g0647701 & Zinc finger $\mathrm{CCCH}$ domain-containing protein 38 & K687Q & -29 \\
\hline Solyc10g0804101 & BZIP transcription factor & T4031 & -4147 \\
\hline \multicolumn{4}{|c|}{ Transcription regulators } \\
\hline Solyc07g0519801 & Chromodomain-helicase-DNA-binding protein 1-like & A618T & -2833 \\
\hline Solyc09g0760102 & PHD finger family protein & Y390C/L988F & $-3167 /-6472$ \\
\hline \multicolumn{4}{|l|}{ Cell wall } \\
\hline Solyc02g0782301 & Glucan synthase like 1 & V1659G & -5286 \\
\hline Solyc05g0518702 & Pollen allergen Phl p 11 & A134T & -3344 \\
\hline Solyc05g0554901 & Laccase-22 & $\mathrm{R} 472 \mathrm{H}$ & -49 \\
\hline \multicolumn{4}{|l|}{ RSV } \\
\hline Gene & Annotation & Variant & Score value \\
\hline \multicolumn{4}{|l|}{ Transcription factors } \\
\hline Solyc01g1038301 & Zinc finger-homeodomain protein 2 & R56Q & -4000 \\
\hline Solyc03g0971202 & Heat stress transcription factor $\mathrm{A} 3$ & W469L & $-4,548$ \\
\hline Solyc07g0550001 & Myb-related transcription factor & T110l & -5433 \\
\hline Solyc08g0052902 & BZIP transcription factor 3 & E357G & -5548 \\
\hline Solyc08g0062401 & B3 domain-containing protein & R299W & -4223 \\
\hline Solyc09g0080402 & RNA polymerase sigma-70 factor & G436R & -5900 \\
\hline \multicolumn{4}{|c|}{ Transcription regulators } \\
\hline Solyc01g0497401 & DNA repair and recombination protein RAD54-like & R155T & -4767 \\
\hline Solyc02g0646902 & Acetyltransferase-like protein & $112 \mathrm{~T}$ & -3386 \\
\hline \multicolumn{4}{|l|}{ Cell wall } \\
\hline Solyc01g0087201 & Mannan endo-1 4-beta-mannosidase & G215S & -5733 \\
\hline Solyc03g1153101 & Expansin & E146G & -3595 \\
\hline Solyc11g0565901 & Cellulose synthase & D100N & -4472 \\
\hline \multicolumn{4}{|l|}{ Common } \\
\hline Gene & Annotation & Variant & Score value \\
\hline \multicolumn{4}{|l|}{ Transcription factors } \\
\hline Solyc00g1983601 & Zinc finger $\mathrm{CCCH}$ domain-containing protein 30 & K188T & -4865 \\
\hline Solyc02g0773901 & WUSCHEL-related homeobox 11 & T291K & -2933 \\
\hline Solyc02g0815202 & Nibrin & F236L & -5433 \\
\hline Solyc03g0439102 & BSD domain containing protein & G54C & -8114 \\
\hline Solyc05g0502202 & G-box binding factor 3 & N335S & -4567 \\
\hline Solyc07g0418501 & Homeodomain-containing transcription factor FWA & 1545T & -4584 \\
\hline Solyc09g0075502 & Zinc finger family protein & V111L & -3000 \\
\hline Solyc09g0075701 & MYB transcription factor & K112I & -6351 \\
\hline Solyc10g0181101 & MADS box transcription factor & L104P & -3739 \\
\hline Solyc12g0107601 & Transcription factor (Fragment) & $\mathrm{A} 36 \mathrm{~V} / \mathrm{G} 44 \mathrm{C} / \mathrm{L} 38 \mathrm{P}$ & $-2844 /-7506 /-6344$ \\
\hline Solyc12g0198201 & MYB transcription factor & K14R & -2942 \\
\hline
\end{tabular}


Table 8 Fruit quality related genes affected by deleterious mutation in SM, RSV or both varieties (Continued)

\begin{tabular}{llll}
\hline $\begin{array}{l}\text { Transcription regulators } \\
\text { Solyc04g0086102 }\end{array}$ & Histone acetyltransferase & P1416L & -7522 \\
Solyc06g0842502 & Kelch repeat and BTB domain-containing & A324T & -2700 \\
Solyc08g0687701 & N-acetyltransferase & W198L & -12149 \\
Solyc10g0062202 & Cell differentiation protein rcd1 & Q215E & -2957 \\
Ripening ethylene related & & & -2612 \\
Solyc01g0791802 & Pectinesterase & T334l & -4364 \\
Solyc01g0811801 & Beta-glucosidase & H83Y & -3582 \\
Solyc01g0915302 & Fasciclin-like arabinogalactan protein 13 & S201Y & -5337 \\
Solyc02g0676501 & Polygalacturonase 1 & G320S & -3776 \\
Solyc05g0500102 & 1-aminocyclopropane-1-carboxylate synthase & T82A & $-3085 /-4045$ \\
Solyc05g0525301 & Endoglucanase 1 & A183V/197T & -2583 \\
Solyc08g0653202 & Transmembrane protein 222 & V157L & -5217 \\
Solyc09g0076501 & Fasciclin-like arabinogalactan protein 7 & S104F & -3709 \\
Solyc09g0102102 & Endoglucanase 1 & A306V & \\
\hline
\end{tabular}

Data are referred to non-synonymous amino acid variants in CDS regions PROVEAN score threshold $=-25$ (Variants with score equal or below -25 are considered "deleterious").

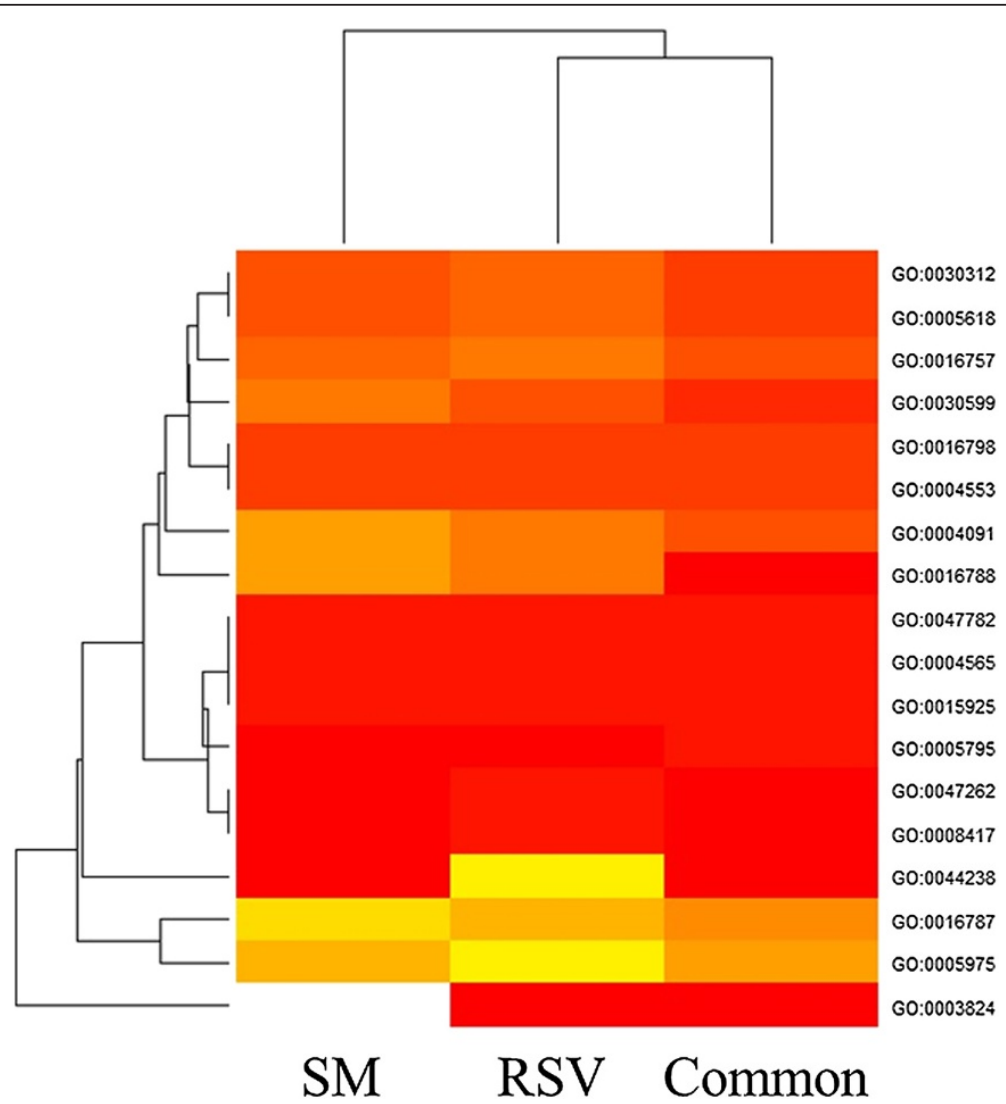

Figure 5 GO terms enrichment heat map. Significant GO term classes enriched Vesuviano (RSV) and San Marzano (SM) non-synonymous coding variants are reported Variants found in both varieties respect reference genome are indicated as common; variants found in each variety (RSV or SM) are indicated as specific. 
Analysis of genetic variants for quality related genes showed that genes were differentially affected by genetic variants depending on the functional class they belong to, suggesting different degrees of selection for genetic variants underlying biological processes. We also showed that the position of sequence variants influence the functionality of the encoded protein. Functional variants contributing to deletion in 3'UTR and exon, intron_conserved and exon, intron_conserved region were highlighted; by contrast, a limited number of other intronic/esonic variants were identified. SNPs within the gene classes assessed reflect the fruit quality genetic diversity between RSV and SM varieties. High percent of variation and deleterious substitutions has been found in genes belonging to the transcription factor and transcription regulator classes, such as acetyltrasferase, chromodomain helicase and histone linkers. Interestingly, enrichment for a chromatin remodeler like protein ligase SNF2 in RSV genotypes points out the possibility that the phenotypic differences among these three tomato genotype are mainly due to complex mechanisms of gene regulation and cross-talk. Recently it has been showed that important epigenome modifications are associated with ripening process [35]. The ethylene-related gene class also showed a high number of variants and deleterious substitutions, probably due to the large difference in the ripening process of the two tomato varieties with respect to the reference tomato. In particular, an ACS gene showed a deleterious substitution (T82A) in both varieties and three ACS key genes involved in ethylene biosynthesis varied only in RSV. This is a long-storage tomato variety with extended shelf life. Since ethylene control fruit ripening process [36], polymorphisms detected in these RSV genes should be further explored to understand their involvement in delaying ripening process. Ethylene production is regulated by combinatorial interplay of the ACS polypeptides. Understand how the "ACS symphony orchestra" is coordinated will be a big challenge for the future [37]. Finally, the result of SEA analyse indicated a significant enrichment of the cell wall genes. GO terms corresponding to hydrolase, galactosidase, beta-glucosidase and beta-galactosidase, involved in chemicals breakdown activity inside the fruits, showed significant differences in both varieties. In particular, RSV-specific non-synonymous variants were enriched in genes involved in xyloglucan biosynthesis and homogalacturonan biosynthesis. Genes related to fruit texture has been frequent targets for genetic engineering, with the goal of extending shelf life [38]. Future investigations on these genes and ethylene related genes should be achieved. The regulation of texture and shelf life is complex and performing a deeper analysis of variants discovered could allow a better understanding of the relationship between changes in the textural and shelf life extension [36].

\section{Conclusions}

The genome sequences reported here and the variants catalogue obtained will be useful to identify the molecular basis of gene complex patterns. Further analysis and functional studies will serve as a basis for understanding trait differences, which will facilitate the identification of markers for genomic marker-assisted breeding. Data produced can be also useful to prioritize mutations to reveal a phenotype. Indeed, large-scale TILLING projects can be used to identify gene of interest saturated with mutations [39]. Collectively, sequence and fine annotation analysis performed can be useful to examine the path from sequence variant to phenotype for improving the utility of the tomato as a model for fruit quality. In addition, the genes we identified that are related to ripening and texture characteristics could be used as target for tomato breeding. The local genomes genotyping is also useful for understanding the genomic features that distinguish modern from traditional varieties. Variants specific for SM and RSV might be explored through a high throughput target re-sequencing approach in other varieties in order to verify that they could represent variants characteristics for these two different tomato typologies.

\section{Additional files}

Additional file 1: Figure S1. Fruit morphological characteristics.

Additional file 2: Table S1. Sequencing statistics. Statistics of sequencing of Vesuviano (RSV) and San Marzano (SM) cultivars of Solanum lycopersicon. Table S2. RepeatMasker results statistics. SL2.40 genome is annotated with similar number of mobile elements (63\%) of and with similar outstanding proportion of LTR. Table S3. Genome reconstruction statistics. Size of the reconstructed chromosomes in San Marzano (SM) and Vesuviano (RSV) respect to Heinz SL2.40 (H) Genome. Table S4. Polymorphic regions. General statistics of polymorphic regions detected in RSV and SM genotypes. Table S5. Gene Annotation Transfer. The gene annotation was transferred from the reference to the reconstructed cultivar genome on the basis of the putative effect of called mutations. If the mutation had a putative disrupting effect on the CDS the gene was not transferred to the new annotation. Table S6. List of varied genes in fruit quality and ripening-related classes. Table S7. Non-synonymous exon variants in fruit quality and ripening-related gene classes.

Additional file 3: Figure S2. Assembly accuracy. Assembly accuracy (y axis; number of base substitution errors) at 12 stages of the iterative mapping against the reference genome.

Additional file 4: Figure S3. Distribution of San Marzano (SM) chromosome 9 SNPs density.

Competing interests

The authors declare that they have no competing interests.

\section{Authors' contributions}

EMR was involved in conception and design of study, in interpretation of data and in manuscript writing, SA in gene variants analysis, interpretation of data and in manuscript drafting, FF in acquisition of data and analysis, DR in 
gene variants analysis and writing, TP in gene annotation process and in writing, TA in CDS mapping analysis and writing, BA in gene variants data interpretation and in manuscript drafting, ZE in sequencing and analysis of data, CML analysis, interpretation of data and in manuscript writing, BG in genome assembling and analysis, DM in experiments design and in critically revision of manuscript; FL conceived the study and coordinated the project and has given final approval of the version to be published. All authors read and approved the final manuscript.

\section{Acknowledgements}

We sincerely acknowledge Dr. Giuseppe Andolfo for R programming support and Dr. Alberto Ferrarini for his valuable suggestions in improving the manuscript. We wish to dedicate our effort in memory of Dr. Tina Mancuso.

\section{Funding}

This work was supported by the Ministry of University and Research (GenoPOM-PRO Project), La Semiorto Sementi S.r.l., Sarno, Italy, and Lodato Gennaro \& C. S.P.A., Castel San Giorgio Italy.

\section{Author details}

${ }^{1}$ Department of Agriculture Sciences, University of Naples Federico II, Via Universita' 100, 80055 Portici, Italy. ${ }^{2}$ Dipartimento di Biotecnologie, Università degli Studi di Verona, Strada le Grazie, 15, 37134 Verona, Italy. ${ }^{3}$ Present address: East Malling Research, New Road, East Malling, Kent ME19 6BJ, UK.

Received: 24 July 2013 Accepted: 24 January 2014

Published: 18 February 2014

\section{References}

1. Bai $Y$, Lindhout P: Domestication and breeding of tomatoes: what have we gained and what can we gain in the future? Ann Bot-London 2007, 100:1085-1094.

2. Grandillo S, Chetelat R, Knapp SK, Spooner DM, Peralta I, Cammareri M, Perez O, Termolino P, Tripode P, Chiusano ML, Ercolano MR, Fruschiante L, Monte L, Pignone D: Solanum section Lycopersicon. In Wild crop relatives: Genomic and breeding resources, Volume 5. Edited by Kole C. Berlin, Heidelberg: Springer-Verlag; 2011:129-216.

3. Zago F: Buone varietà di pomodoro. L'Italia Agricola 1912, 44:110-456.

4. Zago F: Varietà per la preparazione dei pelati. L'ttalia Agricola 1929, 66:360-362.

5. Ercolano MR, Carli P, Soria A, Cascone A, Fogliano V, Frusciante L, Barone A Biochemical sensorial and genomic profiling of Italian tomato traditional varieties. Euphytica 2008, 164:571-582.

6. Carli P, Barone A, Fogliano V, Frusciante L, Ercolano MR: Dissection of genetic and environmental factors involved in tomato organoleptic quality. BMC Plant Biol 2011, 11:58.

7. Garcia-Martinez S, Corrado G, Ruiz-Martinez JJ, Rao R: Diversity and structure of a sample of traditional Italian and Spanish tomato accessions. Genet Resour Crop Evol 2013, 60:789-798.

8. The Tomato Genome Consortium: The tomato genome sequence provides insights into fleshy fruit evolution. Nature 2012, 485:635-641.

9. Todd PM, Alba R: The tomato genome fleshed out. Nat Biotechnol 2012, 30:765-767.

10. The 1000 Genomes Project Consortium: An integrated map of genetic variation from 1092 human genomes. Nature 2012, 491:56-65.

11. Cao J, Schneeberger K, Ossowski S, Günther T, Bender S, Fitz1 J, Koenig D, Lanz C, Stegle O, Lippert C, Wang X, Ott F, Müller J, Alonso-Blanco C, Borgwardt K, Schmid KJ, Weigel D: Whole-genome sequencing of multiple Arabidopsis thaliana populations. Nat Genet 2011, 43:956-963.

12. Treangen TJ, Salzberg SL: Repetitive DNA and next-generation sequencing: computational challenges and solutions. Nat Rev Genet 2011, 13:36-46.

13. Schneeberger K, Ossowski S, Ott F, Klein JD, Wang X, Lanz C, Smith LM, Cao J, Fitz J, Warthmann N, Henz SR, Huson DH, Weigel D: Reference-guided assembly of four diverse Arabidopsis thaliana genomes. Proc Natl Acad Sci USA 2011, 108:10249-10254.

14. Gan X, Stegle O, Behr J, Steffen JG, Drewe P, Hildebrand KL, Lyngsoe R, Schultheiss SJ, Osborne EJ, Sreedharan VT, Kahles A, Bohnert R, Jean G, Derwent $P$, Kersey P, Belfield EJ, Harberd NP, Kemen E, Toomajian C, Kover PX, Clark RM, Rätsch G, Mott R: Multiple reference genomes and transcriptomes for Arabidopsis thaliana. Nature 2011, 477:419-423.

15. Bevan M: Genomics: endless variation most beautiful. Nature 2011 477:415-416.

16. Jiménez-Gómez JM, Maloof JN: Sequence diversity in three tomato species: SNPs, markers, and molecular evolution. BMC Plant Biol 2009, 9:85.

17. Lunter G, Goodson M: Stampy: a statistical algorithm for sensitive and fast mapping of Illumina sequence reads. Genome Res 2011, 21:936-939.

18. Gremme G, Brendel V, Sparks ME, Kurtz S: Engineering a software tool for gene structure prediction in higher organisms. Inf Softw Technol 2005, 47:965-978.

19. Krzywinski M, Schein J, Birol I, Connors J, Gascoyne R, Horsman D, Jones S, Marra M: Circos: an information aesthetic for comparative genomics. Genome Res 2009, 19:1639-1645.

20. Cingolani P, Platts A, Wang LL, Coon M, Nguyen T, Wang L, Land SJ, Lu X, Ruden DM: A program for annotating and predicting the effects of single nucleotide polymorphisms, SnpEff: SNPs in the genome of_Drosophila melanogaster strain w1118; iso-2; iso-3. Fly 2012, 6:80-92.

21. Choi Y, Sims GE, Murphy S, Miller JR, Chan AP: Predicting the functional effect of amino acid substitutions and indels. PLOSOne 2012, 7:e46688.

22. Li H, Handsaker B, Wysoker A, Fennell T, Ruan J, Homer N, Marth G, Abecasis G, Durbin R, and 1000 Genome Project Data Processing Subgroup: The Sequence alignment/map (SAM) format and SAMtools. Bioinformatics 2009, 25:2078-2079.

23. Quinlan AR, Hall IM: BEDTools: a flexible suite of utilities for comparing genomic features. Bioinformatics 2010, 26(6):841-842.

24. Ashburner M, Ball CA, Blake JA, Botstein D, Butler H, Cherry JM, Davis AP, Dolinski K, Dwight SS, Eppig JT, Harris MA, Hill DP, Issel-Tarver L, Kasarskis A, Lewis S, Matese JC, Richardson JE, Ringwald M, Rubin GM, Sherlock G: Gene ontology: tool for the unification of biology The Gene Ontology Consortium. Nat Genet 2000, 25:25-29.

25. Conesa A, Götz S, García-Gómez JM, Teroll J, Talónl M, Roblesl M: Blast2GO: a universal tool for annotation visualization and analysis in functional genomics research. Bioinformatics 2005, 21:3674-3676.

26. Du Z, Zhou X, Ling Y, Zhang Z, Su Z: agriGO: a GO analysis toolkit for the agricultural community. Nucl Acids Res 2012, 38:W64-W70.

27. Tipney $H$, Hunter $L$ : An introduction to effective use of enrichment analysis software. Hum Genomics 2010, 4:202-206.

28. Hamilton JP, Sim SC, Stoffel K: Single nucleotide polymorphism discovery in cultivated tomato via sequencing by synthesis. The Plant Genome 2012, 5:17-29.

29. Sim SC, Robbins MD, Van Deynze A, Michel AP, Francis DM: Population structure and genetic differentiation associated with breeding history and selection in tomato (Solanum lycopersicum L). Heredity 2011, 106:927-935.

30. Sim SC, Van Deynze A, Stoffel K, Douches DS, Zarka D, Ganal MW, Chetelat RT, Hutton SF, Scott JW, Gardner RG, Panthee DR, Mutschler M, Myers JR, Francis DM: High-density SNP genotyping of tomato (Solanum lycopersicum $\mathrm{L}$ ) reveals patterns of genetic variation due to breeding. PLOSOne 2012, 7:e45520.

31. Blanca J, Cañizares J, Cordero L, Pascual L, Diez MJ, Nuez F: Variation revealed by SNP genotyping and morphology provides insight into the origin of the tomato. PLOSOne 2012, 7:e48198.

32. Hirakawa H, Shirasawa K, Ohyama A, Fukuoka H, Aoki K, Rothan C, Sato S, Isobe S, Tabata S: Genome-wide SNP genotyping to infer the effects on Gene functions in tomato. DNA Res 2013, 20(3):221-233. doi:10.1093/ dnares/dst005.

33. McHale LK, Haun WJ, Xu WW, Bhaskar PB, Anderson JE, Hyten DL, Gerhardt DJ, Jeddeloh JA, Stupar RM: Structural variants in the soybean genome localize to clusters of biotic stress-response genes. Plant Physiol 2012, 159:1295-1308.

34. Pavlidis $P$, Metzler $D$, Stephan W: Selective sweeps in multilocus models of quantitative traits. Genetics 2012, 192:225-239.

35. Zhong S, Fei Z, Chen YR, Zheng Y, Huang M, Vrebalov J, McQuinn R, Gapper N, Liu B, Xiang J, Shao Y, Giovannoni JJ: Single-base resolution methylomes of tomato fruit development reveal epigenome modifications associated with ripening. Nat Biotechnol 2013, 31:154-159.

36. Klee HJ, Giovannoni JJ: Genetics and control of tomato fruit ripening and quality attributes. Annu Rev Genet 2011, 45:41-59. 
37. Tsuchisaka A, Yu G, Jin H, Alonso JM, Ecker JR, Zhang X, Gao S, Theologis A: A combinatorial interplay among the 1-aminocyclopropane-1-carboxylate isoforms regulates ethylene biosynthesis in Arabidopsis thaliana. Genetics 2009, 183:979-1003.

38. Matas A, Gapper N, Chung M, Giovannoni J, Rose JKC: Biology and genetic engineering of fruit maturation for enhanced quality and shelf-life. Curr Opin Biotech 2009, 20:197-203.

39. Ng PC, Henikoff S: Predicting the effects of amino acid substitutions on protein function. Annu Rev Genomics Hum Genet 2006, 7:61-80.

doi:10.1186/1471-2164-15-138

Cite this article as: Ercolano et al:: Patchwork sequencing of tomato San

Marzano and Vesuviano varieties highlights genome-wide variations.

BMC Genomics 2014 15:138.

\section{Submit your next manuscript to BioMed Central and take full advantage of:}

- Convenient online submission

- Thorough peer review

- No space constraints or color figure charges

- Immediate publication on acceptance

- Inclusion in PubMed, CAS, Scopus and Google Scholar

- Research which is freely available for redistribution 